\title{
Finger Movement Discrimination of EMG Signals Towards Improved Prosthetic Control using TFD
}

\author{
E.F. Shair ${ }^{1 *}$, N.A. Jamaluddin ${ }^{2}$, A.R. Abdullah ${ }^{3}$ \\ Centre of Excellence for Robotic, and Industrial Automation (CERIA) \\ Department of Electrical Engineering, Faculty of Electrical Engineering \\ Universiti Teknikal Malaysia Melaka, Hang Tuah Jaya, Durian Tunggal, 76100 Melaka, Malaysia
}

\begin{abstract}
Prosthetic is an artificially made as a substitute or replacement for missing part of a body. The function of the missing body part can be replaced by using the prosthesis and it can help disabled people do their activities easily. A myoelectric control system is a fundamental part of modern prostheses. The electromyogram (EMG) signals are used in this system to control the prosthesis movements by taking it from a person's muscle. The problem for the myoelectric control system is when it did not receive the same attention to control fingers due to more dexterous of individual and combined finger control in a signal. Thus, a method to solve the problem of the myoelectric control system by using time-frequency distribution (TFD) is proposed in this paper. The EMG features of the individual and combine finger movements for ten subjects and ten different movements is extracted using TFD, ie. spectrogram. Three machine learning algorithms which are Support Vector Machine (SVM), k-Nearest Neighbor (KNN) and Ensemble Classifier are then used to classify the individuals and combine finger movement based on the extracted EMG feature from the spectrogram. The performance of the proposed method is then verified using classification accuracy. Based on the results, the overall accuracy for the classification is $90 \%$ (SVM), $100 \%$ (KNN) and $100 \%$ (Ensemble Classifier), respectively. The finding of the study could serve as an insight to improve the conventional prosthetic control strategies.
\end{abstract}

Keywords-Electromyography; feature extraction; timefrequency distribution; spectrogram; classification; machine learning

\section{INTRODUCTION}

Nowadays, the world just not depends on current science and medicine but instead it also creates a variety of new technologies. Among the attention of the world is the creation of electronic tools that assist in physiotherapy facilities. In the field of physiotherapy, many tools can help to guide paralyzed or disabled patients during rehabilitation training such as prosthetic hand and leg.

Furthermore, the first prosthetic hand and leg which is used for treatments, especially in physiotherapy, was introduced in the early 16th century. Prosthetic is an artificial made as a substitute or replacement for missing part of the body due to accident or permanent disablement. This prosthetic can help disabled people to do their work or activities easily. Our body uses the muscles to control the limb movement. However, in prosthetic, electromyogram (EMG) signals from an individual muscle are used instead [1].
The EMG signals are to record the electrical activity of muscles. This signal knows the condition of muscles and nerve of the body when movements exist. However, the EMG signals can be affected by several factors, especially during data collection [2]. Thus, several methods can be used to get better accuracy of surface EMG signals for a prosthetic hand.

Currently, there is a high technology that can create and manufacture prosthetics which is used to replace the loss part of the body and being normal again. Nowadays, artificial limbs have advancements in the materials used and the design of artificial. There is for enhancements and comfortable use when using the prosthetic. Also, the electronics have been used as new materials and become common in artificial limbs. The myoelectric limbs have become more common than cable operated limbs to control the limbs. The myoelectric has been used by converting the muscle movements to electrical signals. The myoelectric used the electrodes to convert the signals of muscle movements to electrical signals. However, there still have some technical problems in the process of capturing or analyzing the data [3]. The myoelectric signals can be triggered by internal and external disturbances.

Recent attempts have been made to obtain more dexterous human finger power, given the success of using EMG signals in interpreting the expected forearm gestures. For example, using surface EMG signals to determine when the finger is active and which finger is enabled using only two electrodes positioned on the forearm. There was an experiment that used two electrodes to detect four finger movements by using time distribution and neural networks of good accuracy. However, the performance of the time distribution (TD) features are not satisfactory even though time consumption and dimensions of TD is faster and smaller [4]. On the other hand, frequency distribution (FD) features can be difficult to detect EMG signals for stroke subjects due to the lower power frequency at muscle contraction [5].

Besides that, the classification of individual single finger movement is common but there are only several types of research that have been made for the classification of multiple individuals and combine finger movement in the same finger. To recognize the EMG signals from different classes of the finger movements, a suitable classifier must be employed in the system.

*Corresponding Author 


\section{RELATED WORKS}

\section{A. The Problem of Prosthetic Hand}

In the field of medicine research, the necessary function can be continuing by the prosthesis. This new technology and behaviors in the field of a prosthesis can be the modern treatment of diseases such as diabetes, stroke, and peripheral artery disorder. The patient continues to be the final common denominator. The prosthetic is made to replace the function of missing limbs either to walk or moving depending on their desire.

There have a limited performance for prosthetic tools where it is performed with one specific activity or perform by bimanually while the prosthetic hand can perform with multiple activities and tasks. In contrast to the mechanical appearance of prosthetic tools, prosthetic hands appear human-like.

Hybrid devices can be used for high-level amputation for example at or above the elbow with a combination of bodypowered and myoelectric elements. This system can control two joints at once where one is body-powered and the other one is myoelectric. This device is cheaper and smaller than a prosthetic composed entirely of EMG controlled components. However, the myoelectrical prosthetic has a disadvantage where the prosthetic is heavy, expensive than the other type of prosthetic, and depending on usage and power consumption to operate. Other than that, prosthetic, not $100 \%$ reliable because the EMG sensor sometimes gets "misreads" the user intent when it attached to the skin. Currently, the prosthetic on the market is not a full feedback loop and the input proprioceptive sensor is not fed back to the natural neural pathways of the user [6].

\section{B. EMG Signals}

Electromyogram (EMG) is the electrical activity to define nerve and muscle problems in response to a simulation of the muscle of nerve. During the test, a small needle (electrodes) are used to pick up the electrical activity through the skin into muscle and displayed on a monitor in a waveform. The EMG measured the muscle during rest, slight contraction, and forceful contraction for the electrical activity. There are two methods to measure EMG signals: invasive and noninvasive. For invasive methods, it uses needle electrodes while a noninvasive method uses electrodes above the skin surface of the patient's body [7].

EMG is an analytical technique involved in the development, recording, and study of myoelectric signals. Myoelectric signals are formed by physiological changes in the state of the membranes of muscle fiber [8]. EMG signals have a wide range of applications in biomedical engineering and it is one of the vital biological parameters, prosthetic devices, and rehabilitation devices [9]. It is a bio-potential signal acquired through the muscle fiber body by electrodes to analyze muscle activity [9] and these signals measure the electrical activity during contraction and relaxation phase of the muscle fiber [7].

The EMG has also been used to find the effect of symptoms such as muscle weakness, deformity, stiffness, and shrinkage. Other than that, EMG is also used to test the problem of the motor like involuntary muscle twitching and nerve compression, injuries such as carpal tunnel syndrome, injured nerve root, and muscle degeneration.

\section{Feature Extraction}

Features extraction is a significant way of collecting useful information contained in the surface EMG signals and eliminating unnecessary sections and interferences. The features of EMG signals are divided into three groups which are time-domain, frequency domain, and time-frequency domain [10]. The advantages and disadvantages of the features are shown in in Table I.

Time-frequency analysis is evaluated in time and frequency domain as shown in Fig. 1. Features taken from time-frequency distribution (TFD) should be reduced before being sent to the classifier. To improve the accuracy of the classification, time-frequency distribution feature is proposed to overcome the limitation of TD features [12].

The function of TFD feature is to identify time-varying system properties from the non-stationary system. The TFD has a major problem which is high dimensionality and high resolution of features vectors and to overcome the problem is to reduce the dimensionality of the data [12]. Furthermore, mathematical functions described in the time domain and the frequency domain are commonly used as dimensionality reduction methods for TD features [13]. There are two techniques for dimensionality reduction which are feature projection and feature selection. Features projection techniques attempt to determine the best combination of the original features and create a new feature set that is generally smaller than the original one. For feature selection, it needs to consider a features vector for numerous specific EMG signal classification [12].

Time-frequency also used for EMG signal processing by past research. During the test, the surface myoelectric signal is compressed towards the lower frequency and the frequency of the signal is continuous changes over time. This can classify the surface myoelectric as slow and fast. For slow nonstationary is because of the electrical manifestations and affects the accumulation of metabolites. Next, fast nonstationary is related to the biomechanics of the task. The modification of the frequency content of the signal is affected by the variations in muscle force [14].

Besides that the mathematical techniques have been advanced to solve the problem in signal processing where there are combination methods of state space and statistical decision theory. These techniques happen to a broad class of nonlinear problems and focus on the presence of additive noise due to the problem of signal processing is nonlinear [15].

\section{Classification}

Classification is from the extracted information of the EMG signals to map different patterns and match them appropriately. The classifier is to divide different categories of the features extracted and going to practice being control commands for the controller in the next stage [12]. There are 
many techniques to classify EMG data and have their advantages and disadvantages as shown in Table II.

The problem for biomedical is when there are a few applications that can analyse the demand of patients. So, the application of machine learning can solve this problem with the detection and classification of the neuromuscular disorder based on EMG signal processing. From this application, the patients can skip techniques of ultrasound or MRI to diagnose the neuromuscular disorder. Many biomedical used the support vector machine (SVM) in signal classification applications as a machine learning method. The SVM can improve the accuracy of EMG signal classification and classify it into normal, neurogenic, or myopathic. The classification for SVM is applied based on the trained model after generated the training data in the training process [16].

\section{TABLE I. FEATURE EXTRACTION DOMAIN}

\begin{tabular}{|l|l|l|}
\hline Features Domain & Advantages & Disadvantages \\
\hline Time domain & $\begin{array}{l}\text { Low noise } \\
\text { environments } \\
\text { Lower computational } \\
\text { complexity [10] }\end{array}$ & $\begin{array}{l}\text { Non-stationary property of } \\
\text { EMG signal } \\
\text { Changing in statistical } \\
\text { properties over time [10] }\end{array}$ \\
\hline \multirow{5}{*}{ Frequency domain } & $\begin{array}{l}\text { Reducing interference } \\
\text { Good localization of } \\
\text { the signal. } \\
\text { Very clean signal [11] }\end{array}$ & $\begin{array}{l}\text { High noise environment } \\
{[11]}\end{array}$ \\
\hline $\begin{array}{l}\text { Time-frequency } \\
\text { domain }\end{array}$ & $\begin{array}{l}\text { Can overcome the } \\
\text { limitation of time- } \\
\text { domain features [12] }\end{array}$ & $\begin{array}{l}\text { High dimensionality } \\
\text { High resolution of feature } \\
\text { vectors [12] }\end{array}$ \\
\hline
\end{tabular}

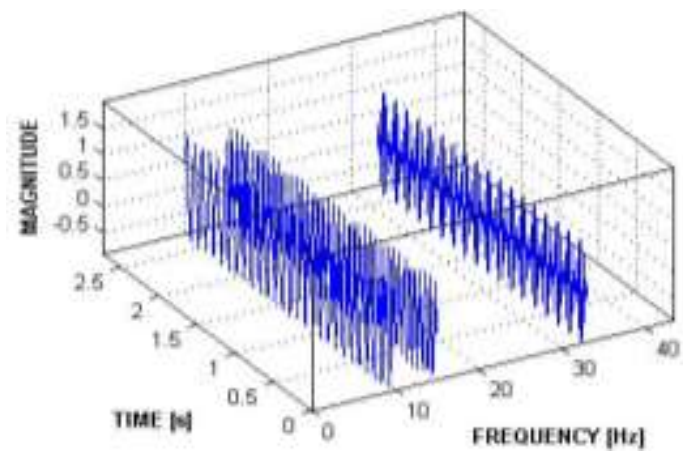

Fig. 1. Graph of Time-Frequency Domain.

TABLE II. CLASSIFICATION TECHNIQUES

\begin{tabular}{|l|l|l|}
\hline $\begin{array}{l}\text { Techniques of } \\
\text { classification }\end{array}$ & Advantages & Categorize \\
\hline $\begin{array}{l}\text { Support vector } \\
\text { machine (SVM) }\end{array}$ & $\begin{array}{l}\text { Works relatively well when } \\
\text { there is a clear margin of } \\
\text { separation between classes }\end{array}$ & Multiple motions \\
\hline $\begin{array}{l}\text { K-nearest neighbor } \\
\text { (KNN) }\end{array}$ & Simple implementation & Hand motion \\
\hline $\begin{array}{l}\text { Multilayer } \\
\text { perceptron (MLP) }\end{array}$ & $\begin{array}{l}\text { Capable of prescribing } \\
\text { nonlinear class boundaries }\end{array}$ & $\begin{array}{l}\text { Hand motion and } \\
\text { forearm motion }\end{array}$ \\
\hline $\begin{array}{l}\text { Artificial neural } \\
\text { networks (ANN) }\end{array}$ & $\begin{array}{l}\text { Suitable for modeling } \\
\text { nonlinear data due can cover } \\
\text { the distinctions }\end{array}$ & $\begin{array}{l}\text { Hand motion (left, } \\
\text { right, up, and down) }\end{array}$ \\
\hline Fuzzy logic (FL) & $\begin{array}{l}\text { Control techniques in } \\
\text { biosignal processing }\end{array}$ & $\begin{array}{l}\text { Biosignal } \\
\text { characteristics }\end{array}$ \\
\hline
\end{tabular}

Recently, k-nearest neighbor (KNN) is a common machine learning tool due to its speed of processing and simplicity in the process of recognition. The concept of $\mathrm{KNN}$ is quite simple. The KNN algorithm creates a set of $\mathrm{k}$ data points in training data and forecasts test data dependent on the nearest neighbor. However, the significance of $\mathrm{k}$ must be carefully chosen because it has a direct effect on the efficiency of the classification. Specifically, the k-value depends mostly on the specification of the data set and model. However, the KNN algorithm is fast, easy, and effective [17].

\section{MATERIALS AND METHODS}

\section{A. EMG Data}

The data for this project is obtained from open source by past research. The data includes EMG signal of finger movement from ten subjects which are six males and four females aged between 20 and 35 years old. The characteristic of the subject is normally limbed with no neurological or muscular disorders. EMG data are taken from this subject by using EMG channels. To firmly stick the sensor to the skin, two of the slot adhesive skin interface (DELSYS DE 2X SERIES EMG SENSOR) was applied to each of the sensors. There are ten classes of finger movements were including the movements for individuals and combined. The duration of every movement is in $5 \mathrm{~s}$ with a resting period in 3 to $5 \mathrm{~s}$ between each movement. The positions of the first electrode are adhesive skin interface stick to the skin and second electrode on the wrist. Positions of the electrode are shown in Fig. 2. Fig. 3 shows the data acquisition set up for the EMG data recording.

\section{B. Signal Pre-Processing}

In the EMG analysis, signal pre-processing which includes eliminating the offset signal, signal segmentation, and detection of onset are required. The signal offset was estimated using the baseline signal means. The offset in each channel was subtracted from the signal to remove the unwanted signal. Segments are calculated to produce signals indicating muscle activation before the features were removed and movement patterns were observed. The auto-segmentation as proposed by [18] was used to segment the EMG signal, thus, helping to reduce the computational complexity of the feature extraction. The magnitude and frequency of muscle activation segments varied from those of muscle activation segments.

The data EMG signals have been filtered in this stage. It is to improve the accuracy of data EMG signals invalidation. Besides, the filtering process is needed to overcome the noise in raw data signals and reduce the artifacts by using the various method. In this stage, the bandpass filter methods have been used to filter the data EMG signals with a range between 20 and $450 \mathrm{~Hz}$. The filtering has been done for separate signals for each movement of EMG signals and to pass the only certain range of frequency and sampling rate is $4000 \mathrm{~Hz}$. The unnecessary noise will be discarded following the range of bandpass filters. However, the noise in signals is very difficult to remove. This stage will give a big impact on classification because the accuracy of signals is depending on the data EMG signals. 


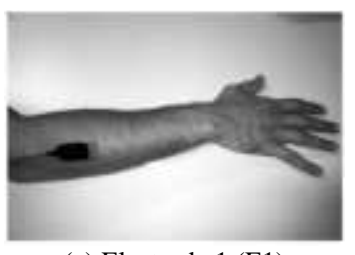

(a) Electrode 1 (E1)

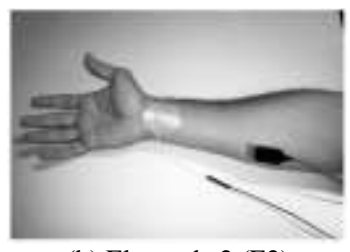

(b) Electrode 2 (E2)
Fig. 2. Electrode Position.

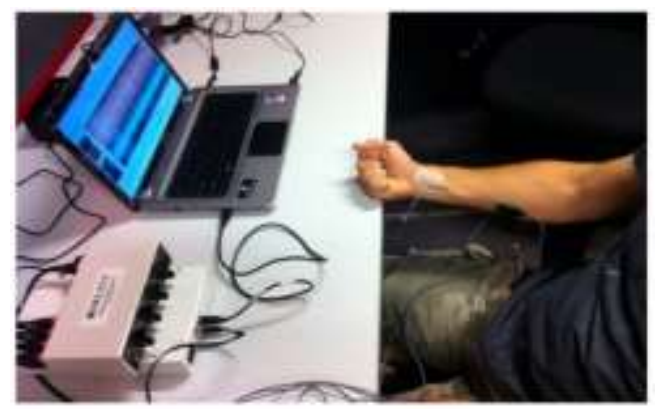

Fig. 3. Data Acquisition Set Up.

\section{Time-Frequency Distribution}

In the field of signal processing, feature extraction plays a critical role throughout achieving better identification quality for the detection of movement patterns. Various signal processing methods are applied in raw EMG to achieve an accurate and actual EMG signal. This process involves the conversions of raw EMG signals into a feature vector. Besides that, the characteristics of the EMG signal analysis can be classified into three groups, which includes the time-domain feature, frequency-domain feature, and time-frequency domain feature. The amplitude of the signals depends on the type and state of the muscle during the analysis phase. Most of the research focuses on time-domain to keep the computational complexity low and this feature does not require additional signal transformation. Various signal processing techniques are used on raw EMG to produce a reliable EMG signal.

In this project, the time-frequency distribution (TFD) which is spectrogram is used in feature extraction. The spectrogram is a fundamental component of TFD in the analysis of signals, particularly for noise and artifact reduction. The spectrogram is used to overcomes the limitation of time and frequency representation for the nonstationary EMG signal. It is defined as the squared magnitude of STFT as expressed in (1).

$S(t, f)=\left|\int_{-\infty}^{\infty} x(\tau) \omega(\tau-t) e^{-j 2 \pi f} d t\right|$

where $S(t, f)$ is the time-frequency representation, $x(\tau)$ is the EMG signal, and $w(t)$ is the observation window.

TFD is preferred to obtain time and frequency information simultaneously. The spectrogram reveals the non-stationary existence of EMG signals in the time-frequency analysis. In TFD, the time and frequency resolution can be adjusted to obtain valuable signal details.

The parameter of the EMG signal was then estimated from the resulted time-frequency representation of the spectrogram.
The root mean square voltage $\left(\mathrm{V}_{\mathrm{rms}}\right)$ was measured instantaneously over time and the average values were taken for hand movement prediction. The average RMS voltage can be expressed as.

$V_{r m s(a v g)}=\frac{1}{T} \int_{0}^{T} V_{r m s}(t) d t$

where

$V_{r m s}(t)=\sqrt{\int_{0}^{f_{\max }} S_{x}(t, f) d f}$

where $V_{\text {rms }}(t)$ is the instantaneous RMS voltage, $S_{x}(t, f)$ is the time-frequency representation, and $f_{\max }$ is the maximum frequency of interest.

\section{Machine Learning}

The information derived from the EMG signals will then be fed into the classifier to identify the different patterns and match them properly. Classifiers should be used to distinguish between different classes of features extracted. The obtained classifications will then be used as control commands for the controller in the next stage. Multiple methods are used to identify EMG information such as artificial neural networks (ANN), Bayesian classifier (BC), fuzzy logic (FL), multilayer perceptron (MLP), support vector machines (SVM), linear discriminant analysis (LDA), hidden Markov models (HMM) and K-nearest neighbor (KNN). Recently, several researchers have shown interest in effective ways to identify the origins of EMG signals.

The machine learning algorithm selected to determine the characteristics of the separation of the 10-finger movement in the EMG data signal after the signal processing phase. In this stage, the data EMG signals of $V_{\text {rms }}$ have been separated according to each movement and electrode by each subject. The total $\mathrm{V}_{\text {rms }}$ data are 200 of 10 subjects with 10 movements for 2 electrodes. The total $\mathrm{V}_{\text {rms }}$ data of EMG signals will be separated into training and testing sets to evaluate the performance of data EMG signals. There are $80 \%$ of data for training and the other $20 \%$ of data for testing. The training test is to train the machine before getting an accurate value for testing. Next, the data have been imported into apps classification learners to analyse the accuracy of classifiers with train the data. There have various types of classifier in classification learner and the wide classifier has been used is KNN and SVM. Therefore, the best selection of classifiers is depending on the percentage of accuracy classifier.

\section{RESUlTS AND DisCUSSIONS}

The EMG signal data consist of ten classes of individual and combined fingers movement. Every subject completed six times of test for 10-finger movements and resting time between the tests is around 3 to 5 seconds. Four of the sixtime test is training and two of that is testing. The raw data is obtained from 10 individual movements which are 5 tests for individual movement and 5 tests for combined movement with two electrodes. This part shows the results and discussions for all methods that have been used.

Results of the EMG signal was obtained from individual finger movement and combine finger movement. The EMG 
signal data was run using the MATLAB software and the graph of the EMG signal is a voltage (V) versus time (s) and it is shown as the amplitude of EMG signal during the test.

There are different finger movements for individuals and combine finger movements. The individual finger movements are consisting of thumb $(\mathrm{T})$, index $(\mathrm{I})$, middle $(\mathrm{M})$, ring $(\mathrm{R})$, little (L). Then, for combined finger movements are consists of hand close (HC), thumb index (T-I), thumb little (T-L), thumb middle (T-M), and thumb ring (T-R). The different movements of the finger are shown as Fig. 4.

These results indicate that using two channels of electrodes during collected data for individual finger movement. It also shows the signal for both electrodes for each movement. From the signals, the electrode 1 is more informative than the electrode 2 for thumb finger movement. This is due to the location of the second electrode where the electrode mounted as shown in Fig. 2. The located of the second electrode is on the low contraction muscle during finger movement because of that the signal of the second electrode not more informative. The informative signal depends on the contraction or muscle movement during the test of the finger movements. The signal of EMG data during the test of thumb movement for the individual finger movement as shown in Fig. 5.

These results show the signal of combine finger movements and indicate two channels of electrodes during the test of finger movement. These signals are made up of two electrodes used during the test of combined finger movements. For $0.7 \mathrm{~s}$ of the signal shows the second electrode gives more information than the first electrode in hand close movement. This is due to the location of electrodes mounted during the test. However, after $0.7 \mathrm{~s}$ the signal of both electrodes shows the same or constants informative. In detail, contraction or muscle movement is higher on the location of the second electrode for $0.7 \mathrm{~s}$, and the muscle movement almost the same after that. The EMG signal for hand close in combined finger movements as shown in Fig. 6.

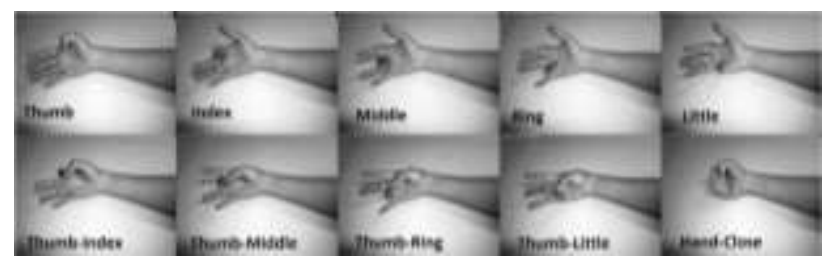

Fig. 4. The different Finger Movements.

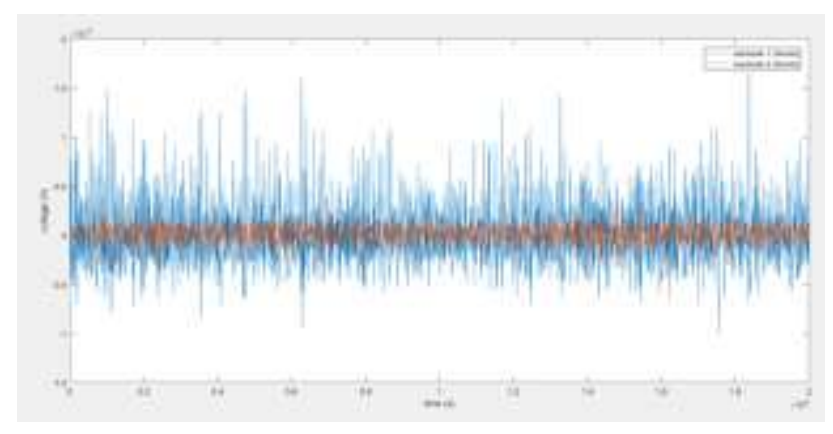

Fig. 5. Raw EMG Signal for Thumb Finger Movement.

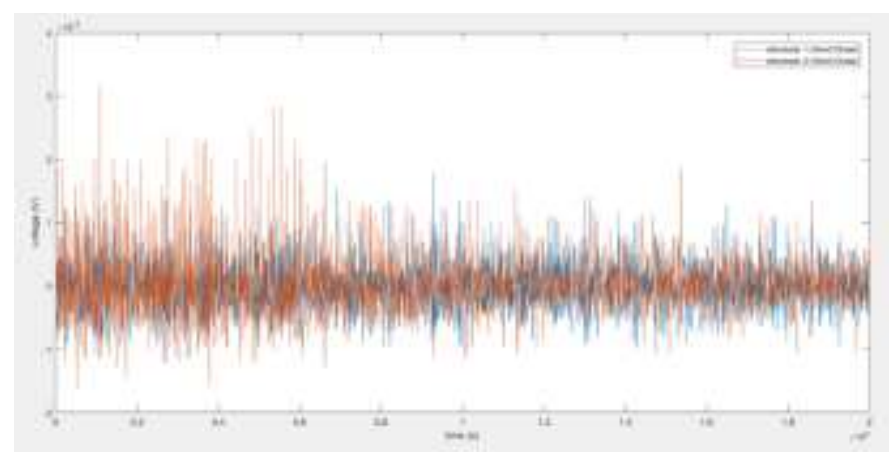

Fig. 6. Raw EMG Signal for Hand Close Finger Movement.

In this part, the signals of EMG are based on the information given on the contraction or muscle movement during the test of finger movement for individual and combined movements. The location of the electrodes is important to collect the data during the test. This EMG signal needs to filter to reduce unnecessary noise before the signal processing stage. The filtering process has been done for each movement and separated by electrodes. The bandpass filter has been used with range $20 \mathrm{~Hz}$ to $450 \mathrm{~Hz}$ and the sampling rate is $4000 \mathrm{~Hz}$. The signal only passes by the range of frequency and the other will discard. The graph shows the EMG signal before and after the filtering process for the middle finger movement for electrode 1 . The filtered middle finger movement as shown in Fig. 7.

In signal processing, the TFD is selected as a fundamental component to analyse the EMG signal, especially for noise. From this, the time and frequency can be measured for RMS. In TFD, to obtain valuable signal details in the EMG signal it can adjust the time and frequency resolution. The extraction of instantaneous RMS voltage EMG signal for electrode 1 of hand close finger movement is shown in Fig. 8.

The average RMS voltage of the EMG signals for individual finger movement is shown in Fig. 9. In the figure, subject 4 shows the highest level for electrode 1 and electrode 2 compare to the other subject in the index finger movement. Thus, during the test of individual finger movement, the subject 4 get more information in signal due to contraction or muscle movement. Subject 4 gives the best signal to control or classify the EMG signal for individual finger movement.

The average RMS voltage of the EMG signals for combine finger movement is shown in Fig. 10. Based on the figure, subject 4 have the highest level of electrode 1 and electrode 2 for average combined finger movement compared to the other subject. From this, the contraction or muscle movement at the location of electrode mounted for subject 4 is higher during testing. The EMG signal for subject 4 has more information about the muscle movement for electrode 1 and electrode 2 in hand close finger movement.

Fig. 11 shows the average $V_{\text {rms }}$ signals for each electrode from all subjects. The average of data EMG signals is to investigate the various levels of acceptance of the suggested. The figure shows the level of information in EMG signals based on the finger movement. The higher level of the graph means the more informative the EMG signals. 


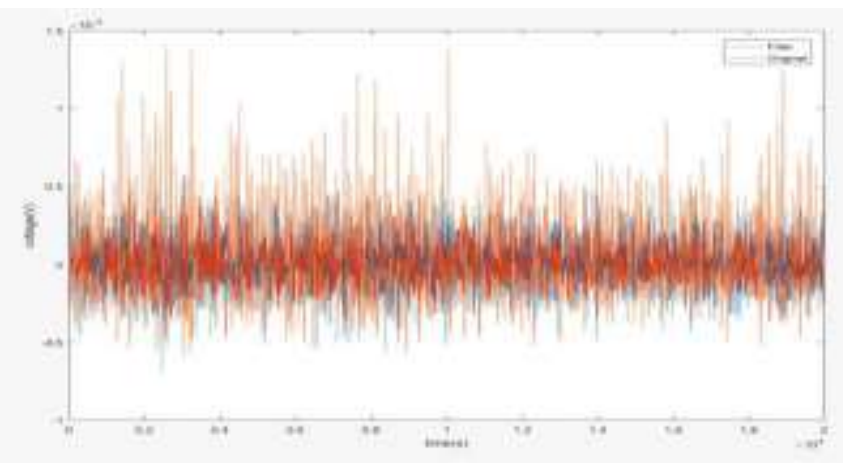

Fig. 7. Raw and Filtered EMG Signal.

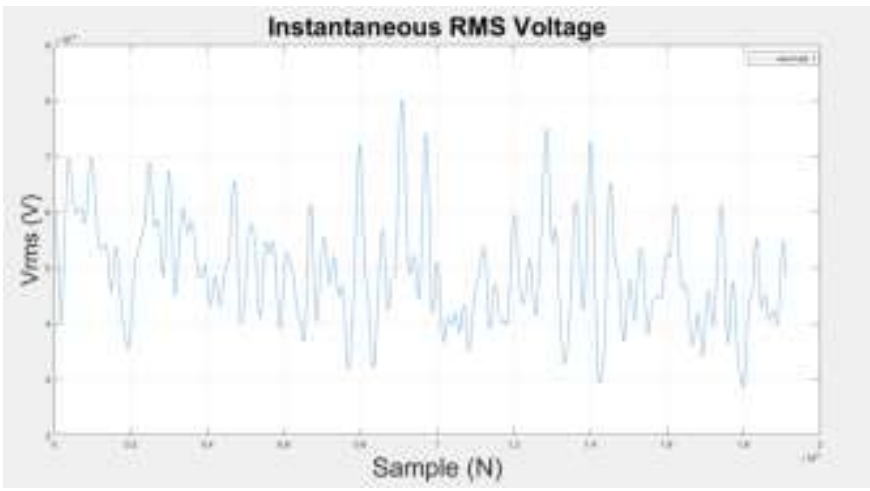

Fig. 8. Instantaneous RMS Voltage for Hand Close Finger Movement.

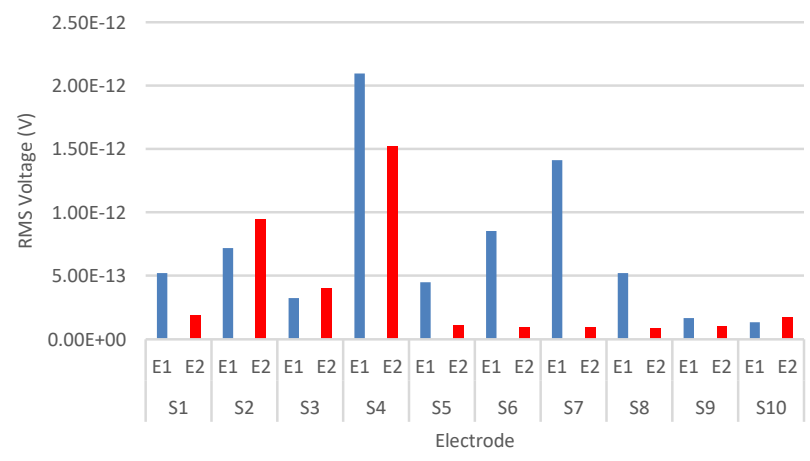

Fig. 9. Average RMS Voltage for Individual Finger Movement (Index).

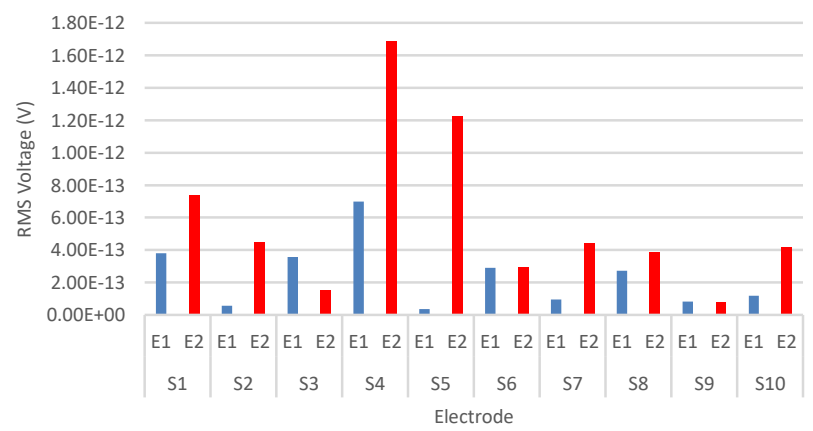

Fig. 10. Average RMS Voltage for Combined Finger Movement (Hand Close).

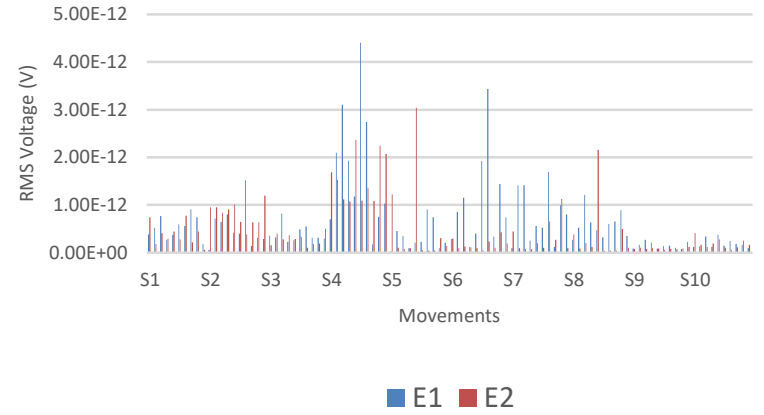

Fig. 11. The Average $V_{\text {rms }}$ EMG Signals for Each Electrode from All Subjects.

The final step in this project is to determine a suitable classifier for data EMG signal from different classes of finger movements. After getting an average of Vrm data for EMG signal, techniques classifiers are used in signal classification to analyse the best machine learning for data EMG signal. The techniques are typically used to avoid confusing the prosthetic controller with different classification decisions and to increase the efficiency of the classifier by avoiding unnecessary classification errors. There is the comparison of techniques classifier for $100 \%$ data EMG signal as shown in Table III.

The total $\mathrm{V}_{\text {rms }}$ data EMG signal will divide into two-part which is $80 \%$ for training and the other $20 \%$ for testing. The training data is to train the machine learning before taking the results for testing finger movements. There is the comparison data for $80 \%$ and $20 \%$ of $\mathrm{V}_{\text {rms }}$ EMG signal as shown in Table IV.

From the comparison results, the best classifier for $\mathrm{V}_{\mathrm{rms}}$ data EMG signal is the k-nearest neighbor $(\mathrm{KNN})$. This is because the percentage of the accuracy of the KNN classifier is $100 \%$ for training and testing which is more accurate from the other classifier. The accuracy of EMG classification is determined based on the percentage in classification learner and can be a plot by a scatter plot and confusion matrix. A scatter plot or scatter graph is displaying the values of two variables from a set of data and identify the type of relationship between variables. The scatter plot for $80 \%$ of $\mathrm{V}_{\text {rms }}$ data EMG signal as shown in Fig. 12 and the scatter plot for $20 \%$ of $\mathrm{V}_{\mathrm{rms}}$ data EMG signal as shown in Fig. 13.

Next, the confusion matrix or table of confusion is showing the error matrix for data with predicted class and actual class. The confusion matrix can plot by true positive rates and false-negative rates. The confusion matrix for $80 \%$ of $\mathrm{V}_{\text {rms }}$ data EMG signal as shown in Fig. 14 and the confusion matrix for $20 \%$ of $\mathrm{V}_{\text {rms }}$ data EMG signal as shown in Fig. 15.

TABLE III. THE COMPARISON FOR 100\% DATA EMG SIGNAL

\begin{tabular}{|l|l|}
\hline Type of classifier & Percentage (\%) \\
\hline SVM (fine gaussian SVM) & $64 \%$ \\
\hline KNN (fine KNN) & $100 \%$ \\
\hline ENSEMBLE CLASSIFIER (boosted trees) & $73 \%$ \\
\hline
\end{tabular}


TABLE IV. THE COMPARISON OF 80\% (TRAINING) AND 20\% (TESTING) OF THE DATA EMG SIGNAL

\begin{tabular}{|l|l|l|}
\hline Classifier & $\mathbf{8 0 \%}$ of data & $\mathbf{2 0 \%}$ of data \\
\hline SVM & $75 \%$ & $90 \%$ \\
\hline KNN & $100 \%$ & $100 \%$ \\
\hline Ensemble Classifier & $73 \%$ & $100 \%$ \\
\hline
\end{tabular}

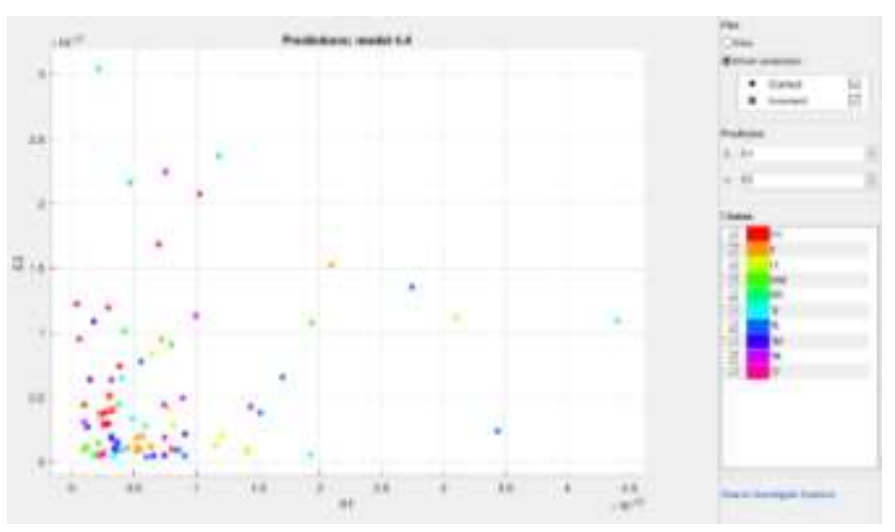

Fig. 12. The Scatter Plot for $80 \%$ of $\mathrm{V}_{\mathrm{rms}}$ Data EMG Signal (Training).

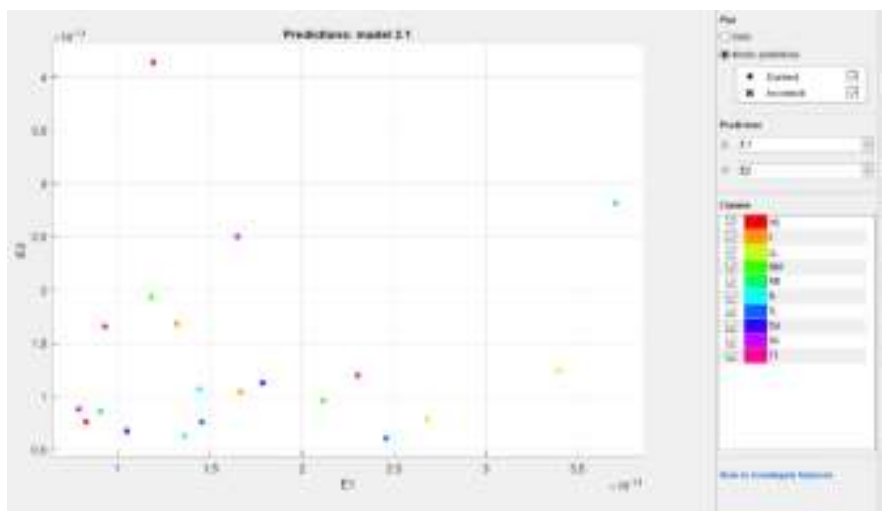

Fig. 13. The Scatter Plot for $20 \%$ of $\mathrm{V}_{\text {rms }}$ Data EMG Signal (Testing).

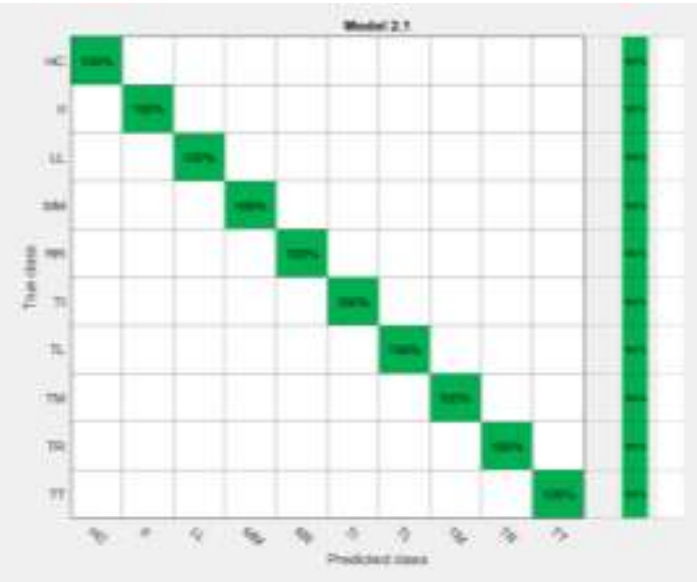

Fig. 14. The Confusion Matrix for $80 \%$ of $\mathrm{V}_{\mathrm{rms}}$ Data EMG Signal (Testing).

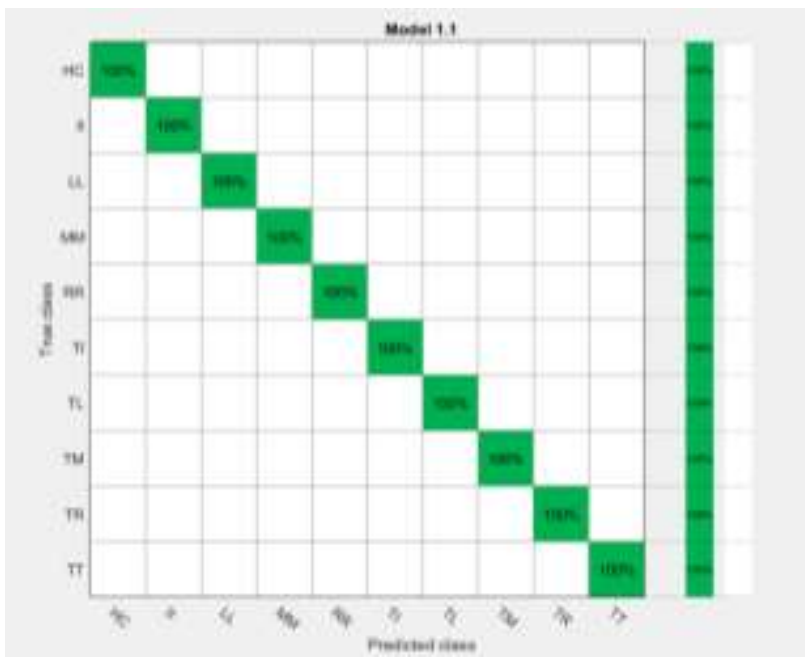

Fig. 15. The Confusion Matric for $20 \%$ of $\mathrm{V}_{\text {rms }}$ Data EMG Signal (Testing).

As a result, significant increases in classification precision have been made. In the signal classification, three classifiers have been used to train the training and testing data of the $\mathrm{V}_{\mathrm{rms}}$ EMG signal. The most accurate for the $\mathrm{V}_{\mathrm{rms}}$ EMG signal is the KNN classifier with $100 \%$ accuracy for training and testing. This is because the KNN classifier is easy to implement.

\section{CONCLUSIONS}

As a conclusion, the research to analyze the surface EMG signals $\left(\mathrm{V}_{\mathrm{rms}}\right)$ in finger movement by using the TFD have been presented. This analysis covered the analysis data of an individual and combined finger movements of EMG signals for prosthetic hand control. The EMG signals have been filtered by using the bandpass filter to overcome the unnecessary noise in signals with a range from 20 to $450 \mathrm{~Hz}$. TFD is then used for the feature extraction to get the average of signal to classification.

In addition, this research classify the accuracy of individual and combine finger movement based on surface EMG signals towards improved prosthetic control. The three classifiers have been used to train all data EMG signal and the most accurate classifier have been chosen as machine learning to conduct the EMG signals. The data of EMG signals have been trained followed by each movement and each subject.

Finally, the performance of the KNN classifier has been compared with other classifiers. The data $\mathrm{V}_{\text {rms }}$ EMG signals have divided into two parts which are training and testing. For instance, the training data is to train the machine learning to get accurate data for testing. The EMG datasets are belong to 10 different classes for individual and combined movements collected from 10 subjects by using two channels of electrodes and the accuracy of classifier in the range $64 \%$ to $100 \%$ with various types of classifiers of the data $\mathrm{V}_{\mathrm{rms}}$ EMG signals. 


\section{FUTURE WORKS}

For future works, further studies about other finger movements for individual and combined finger movements is essential. It is to get data for other movements towards the prosthetic hand. Next, the EMG signal must be tested with prosthetic hand to make sure the classifier that has been chosen is suitable and can be integrated with the prosthetic hand. This is to ensure the accuracy of the classifier is accurate even after intergrating it with the prosthetic hand.

\section{ACKNOWLEDGMENT}

This project is fully funded by Universiti Teknikal Malaysia Melaka under Short Term Grant Scheme (High Impact) no. PJP/2020/FKE/HI19/S01717.

\section{REFERENCES}

[1] S. Pancholi and A. M. Joshi, "Portable EMG Data Acquisition Module for Upper Limb Prosthesis Application," in IEEE Sensors Journal, vol. 18, no. 8, pp. 3436-3443, 15 April15, 2018.

[2] S. Rezazadeh, D. Quintero, N. Divekar and R. D. Gregg, "A Phase Variable Approach to Volitional Control of Powered Knee-Ankle Prostheses," 2018 IEEE/RSJ International Conference on Intelligent Robots and Systems (IROS), Madrid, 2018, pp. 2292-229.

[3] V. K. Mishra, V. Bajaj, A. Kumar and G. K. Singh, "Analysis of ALS and normal EMG signals based on empirical mode decomposition," in IET Science, Measurement \& Technology, vol. 10, no. 8, pp. 963-971, 112016.

[4] E. F. Shair, S. A. Ahmad, M. H. Marhaban, A. R. Abdullah and S. B. M. Tamrin, "Implementation of Spectrogram for an Improved EMG-based Functional Capacity Evaluation's Core-Lifting Task," 2018 IEEEEMBS Conference on Biomedical Engineering and Sciences (IECBES), Sarawak, Malaysia, 2018, pp. 13-17.

[5] R. D. Wilson, S. J. Page, M. Delahant, J. S. Knutson, D. D.Gunzler, L. R. Sheffler and J. Chae, "Upper-Limb Recovery After Stroke: A Randomized Controlled Trial Comparing EMG-Triggered, Cyclic, and Sensory Electrical Stimulation," Neurorehabilitation and Neural Repair, vol. 30, no. 10, 2016, pp. 978-987.

[6] P. Beckerle, S. Willwacher, M. Liarokapis, M. P. Bowers, and M. B. Popovic, "9 - Prosthetic Limbs," M. B. B. T.-B. Popovic, Ed. Academic Press, 2019, pp. 235-278.

[7] J. L. Segil, S. A. Huddle and R. F. f. Weir, "Functional Assessment of a Myoelectric Postural Controller and Multi-Functional Prosthetic Hand by Persons With Trans-Radial Limb Loss," in IEEE Transactions on
Neural Systems and Rehabilitation Engineering, vol. 25, no. 6, pp. 618627, 2017.

[8] P. Konrad, The ABC of EMG - Noraxon, vol. 1, no. March. 2006.

[9] S. Pancholi and A. M. Joshi, "Portable EMG Data Acquisition Module for Upper Limb Prosthesis Application,” IEEE Sens. J., vol. 18, no. 8, pp. 3436-3443, 2018.

[10] D. Zhou, Y. Fang, J. Botzheim, N. Kubota and H. Liu, "Bacterial memetic algorithm based feature selection for surface EMG based hand motion recognition in long-term use," 2016 IEEE Symposium Series on Computational Intelligence (SSCI), Athens, 2016, pp. 1-7.

[11] L. Wu, X. Zhang, X. Chen and X. Chen, "Visualized Evidences for Detecting Novelty in Myoelectric Pattern Recognition using 3D Convolutional Neural Networks*," 2019 41st Annual International Conference of the IEEE Engineering in Medicine and Biology Society (EMBC), Berlin, Germany, 2019, pp. 2641-2644.

[12] N. Nazmi, M. A. A. Rahman, S. I. Yamamoto, S. A. Ahmad, H. Zamzuri, and S. A. Mazlan, "A review of classification techniques of EMG signals during isotonic and isometric contractions," Sensors, vol. 16, no. 8, pp. 1-28, 2016.

[13] S. Inoue, M. Oya and H. Ohta, "Finger Joint Dynamics with Myoelectric Signal inputs," 2018 International Conference on Information and Communication Technology Robotics (ICT-ROBOT), Busan, 2018, pp. $1-4$.

[14] N. Jose, R. Raj, P. K. Adithya and K. S. Sivanadan, "Classification of forearm movements from sEMG time domain features using machine learning algorithms," TENCON 2017 - 2017 IEEE Region 10 Conference, Penang, 2017, pp. 1624-1628.

[15] A. Furui, H. Hayashi, Y. Kurita and T. Tsuji, "Variance distribution analysis of surface EMG signals based on marginal maximum likelihood estimation," 2017 39th Annual International Conference of the IEEE Engineering in Medicine and Biology Society (EMBC), Seogwipo, 2017, pp. 2514-2517.

[16] M. Kilany, E. H. Houssein, A. E. Hassanien and A. Badr, "Hybrid water wave optimization and support vector machine to improve EMG signal classification for neurogenic disorders," 2017 12th International Conference on Computer Engineering and Systems (ICCES), Cairo, 2017, pp. 686-691.

[17] J. Too, A. R. Abdullah, N. M. Saad, N. M. Ali, and T. N. S. T. Zawawi, "Application of Gabor Transform in the Classification of Myoelectric Signal," vol. 17, no. 2, pp. 873-881, 2019.

[18] E. F. Shair, A. R. Abdullah, T. N. S. Tengku Zawawi, S. A. Ahmad and S. Mohamad Saleh, "Auto-Segmentation Analysis of EMG Signal for Lifting Muscle Contraction Activities", J. Telecommun. Electron. Comput. Eng., vol. 8, no. 7, pp. 17-22, 2016. 